\title{
EXISTENCE AND MULTIPLICITY OF SOLUTIONS FOR AN ELLIPTIC EQUATION WITH $p(x)$-GROWTH CONDITIONS
}

\author{
MIHAI MIHĂILESCU \\ Department of Mathematics, University of Craiova, 200585 Craiova, Romania \\ e-mail:mmihailes@yahoo.com
}

(Received 18 November, 2005; accepted 3 June, 2006)

\begin{abstract}
We study a partial differential equation on a bounded domain $\Omega \subset \mathbb{R}^{N}$ with a $p(x)$-growth condition in the divergence operator and we establish the existence of at least two nontrivial weak solutions in the generalized Sobolev space $W_{0}^{1, p(x)}(\Omega)$. Such equations have been derived as models of several physical phenomena. Our proofs rely essentially on critical point theory combined with corresponding variational techniques.
\end{abstract}

2000 Mathematics Subject Classification. 35D05, 35J60, 35J70, 58E05, 68T40, 76A02.

1. Introduction. The study of partial differential equations with $p(x)$-growth conditions has received more and more interest in recent decades. The specific attention accorded to such problems is due to their applications in mathematical physics. More precisely, such equations are used to model phenomena which arise in elastic mechanics or electrorheological fluids. For a general account of the underlying physics and for some technical applications we refer to $[\mathbf{1}, \mathbf{4}, \mathbf{1 4}, \mathbf{1 6}, \mathbf{2 0}, \mathbf{2 1}, \mathbf{2 4}, \mathbf{2 5}]$ and the references therein.

A typical model of an elliptic equation with $p(x)$-growth conditions is

$$
-\operatorname{div}\left(|\nabla u|^{p(x)-2} \nabla u\right)=g(x, u) .
$$

The operator $\operatorname{div}\left(|\nabla u|^{p(x)-2} \nabla u\right)$ is called the $p(x)$-Laplace operator and it is a natural generalization of the $p$-Laplace operator, in which $p(x)=p>1$ is a constant. For this reason the equations studied in the case in which the $p(x)$-Laplace operator is involved are, in general, extensions of $p$-Laplacian problems. (See for example $[3,9,11]$.) However, we point out that such generalizations are not trivial since the $p(x)$-Laplace operator possesses more complicated nonlinearity; for example it is inhomogeneous. We just remember the fact that defining

$$
\lambda_{1}:=\inf _{u \in W_{0}^{1, p(x)}(\Omega) \backslash\{0\}} \frac{\int_{\Omega}|\nabla u|^{p(x)} d x}{\int_{\Omega}|u|^{p(x)} d x}
$$

we often have $\lambda_{1}=0$ for general $p(x)$, and only under some special conditions $\lambda_{1}>0$. (See [12].) 
In this paper we study the existence of solutions for a boundary value problem of the type

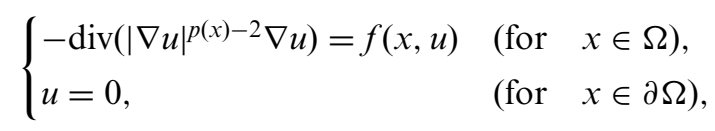

where $\Omega \subset \mathbb{R}^{N}(N \geq 3)$ is a bounded domain with smooth boundary, $1<p(x)$ and $p(x) \in C(\bar{\Omega})$.

Equation (1) will be studied in the framework of the variable Lebesgue and Sobolev spaces $L^{p(x)}$ and $W^{1, p(x)}$ which will be briefly described in the following section.

2. Preliminary results. Variable exponent Lebesgue spaces $L^{p(x)}$, where $p(x)$ is a real-valued function, appeared in the literature for the first time already in a 1931 article by W. Orlicz [19]. In the years 1950 this study was carried on by Nakano [18] who made the first systematic study of spaces with variable exponent. Later, the Polish mathematicians investigated the modular function spaces. (See, for example, Musielak [17].) Variable exponent Lebesgue spaces on the real line have been independently developed by Russian researchers. In this context we refer to the work of Tsenov [23], Sharapudinov [22] and Zhikov [25, 26].

Throughout this paper we assume that $p(x)>1, p(x) \in C(\bar{\Omega})$.

Set

$$
C_{+}(\bar{\Omega})=\{h ; h \in C(\bar{\Omega}), h(x)>1 \text { for all } x \in \bar{\Omega}\} .
$$

For any $h \in C_{+}(\bar{\Omega})$ we define

$$
h^{+}=\sup _{x \in \Omega} h(x) \quad \text { and } \quad h^{-}=\inf _{x \in \Omega} h(x)
$$

For any $p(x) \in C_{+}(\bar{\Omega})$, we define the variable exponent Lebesgue space

$L^{p(x)}(\Omega)=\left\{u ; u\right.$ is a measurable real-valued function such that $\left.\int_{\Omega}|u(x)|^{p(x)} d x<\infty\right\}$

We define a norm, the so-called Luxemburg norm, on this space by the formula

$$
|u|_{p(x)}=\inf \left\{\mu>0 ; \int_{\Omega}\left|\frac{u(x)}{\mu}\right|^{p(x)} d x \leq 1\right\} .
$$

Variable exponent Lebesgue spaces resemble classical Lebesgue spaces in many respects: they are Banach spaces [15, Theorem 2.5], the Hölder inequality holds [15, Theorem 2.1], they are reflexive if and only if $1<p^{-} \leq p^{+}<\infty[\mathbf{1 5}$, Corollary 2.7] and continuous functions are dense if $p^{+}<\infty[15$, Theorem 2.11]. The inclusion between Lebesgue spaces also generalizes naturally [15, Theorem 2.8]: if $0<|\Omega|<\infty$ and $p_{1}$, $p_{2}$ are variable exponent, so that $p_{1}(x) \leq p_{2}(x)$ almost everywhere in $\Omega$, then there exists the continuous embedding $L^{p_{2}(x)}(\Omega) \hookrightarrow L^{p_{1}(x)}(\Omega)$, whose norm does not exceed $|\Omega|+1$. 
We denote by $L^{q(x)}(\Omega)$ the conjugate space of $L^{p(x)}(\Omega)$, where $1 / p(x)+1 / q(x)=1$. For any $u \in L^{p(x)}(\Omega)$ and $v \in L^{q(x)}(\Omega)$ the Hölder type inequality

$$
\left|\int_{\Omega} u v d x\right| \leq\left(\frac{1}{p^{-}}+\frac{1}{q^{-}}\right)|u|_{p(x)}|v|_{q(x)}
$$

holds true.

An important role in manipulating the generalized Lebesgue-Sobolev spaces is played by the modular of the $L^{p(x)}(\Omega)$ space. This is the mapping $\rho_{p(x)}: L^{p(x)}(\Omega) \rightarrow \mathbb{R}$ defined by

$$
\rho_{p(x)}(u)=\int_{\Omega}|u|^{p(x)} d x .
$$

If $u \in L^{p(x)}(\Omega)$ and $p^{+}<\infty$, then the following relations holds true

$$
\begin{gathered}
|u|_{p(x)}>1 \quad \Rightarrow \quad|u|_{p(x)}^{p^{-}} \leq \rho_{p(x)}(u) \leq|u|_{p(x)}^{p^{+}}, \\
|u|_{p(x)}<1 \quad \Rightarrow \quad|u|_{p(x)}^{p^{+}} \leq \rho_{p(x)}(u) \leq|u|_{p(x)}^{p^{-}} \\
|u|_{p(x)}=1 \quad \Rightarrow \quad \rho_{p(x)}(u)=1 .
\end{gathered}
$$

Spaces with $p^{+}=\infty$ have been studied by Edmunds, Lang and Nekvinda [5].

Next, we define $W_{0}^{1, p(x)}(\Omega)$ as the closure of $C_{0}^{\infty}(\Omega)$ under the norm

$$
\|u\|=|\nabla u|_{p(x)} .
$$

The space $\left(W_{0}^{1, p(x)}(\Omega),\|\cdot\|\right)$ is a separable and reflexive Banach space. We note that if $q \in C_{+}(\bar{\Omega})$ and $q(x)<p^{\star}(x)$, for all $x \in \bar{\Omega}$, then the embedding $W_{0}^{1, p(x)}(\Omega) \hookrightarrow L^{q(x)}(\Omega)$ is compact and continuous. Here $p^{\star}(x)=\frac{N p(x)}{N-p(x)}$ if $p(x)<N$ or $p^{\star}(x)=+\infty$ if $p(x) \geq N$. We refer to $[\mathbf{6}, \mathbf{7}, \mathbf{1 0}, \mathbf{1 3}, \mathbf{1 5}]$ for further properties of variable exponent LebesgueSobolev spaces.

3. The main result. In this paper we study problem (1) in the particular case

$$
f(x, t)=A|t|^{a-2} t+B|t|^{b-2} t
$$

with $1<a<p^{-}<p^{+}<b<\min \left\{N, \frac{N p^{-}}{N-p^{-}}\right\}$and $A, B>0$. More precisely, we consider the degenerate boundary value problem

$$
\begin{cases}-\operatorname{div}\left(|\nabla u|^{p(x)-2} \nabla u\right)=A|u|^{a-2} u+B|u|^{b-2} u & (\text { for } x \in \Omega), \\ u=0 & \text { (for } x \in \partial \Omega) .\end{cases}
$$

We seek solutions for problem (6) belonging to the space $W_{0}^{1, p(x)}(\Omega)$ in the sense below.

DEFINITION 1. We say that $u \in W_{0}^{1, p(x)}(\Omega)$ is a weak solution for problem (1) if

$$
\int_{\Omega}|\nabla u|^{p(x)-2} \nabla u \nabla v d x-A \int_{\Omega}|u|^{a-2} u v d x-B \int_{\Omega}|u|^{b-2} u v d x=0,
$$

for all $v \in W_{0}^{1, p(x)}(\Omega)$. 
We prove the following result.

TheOREM 1. There exists $\lambda>0$ such that, for any $A \in(0, \lambda)$ and any $B \in(0, \lambda)$, problem (6) has at least two distinct nontrivial weak solutions.

REMARK. In the case where in equation (6) we assume that $A=B=\mu$ we deal with an eigenvalue problem, namely

$$
\begin{cases}-\operatorname{div}\left(|\nabla u|^{p(x)-2} \nabla u\right)=\mu\left(|u|^{a-2} u+|u|^{b-2} u\right) & (\text { for } x \in \Omega) \\ u=0 & (\text { for } x \in \partial \Omega)\end{cases}
$$

Theorem 1 ensures that problem (7) has a continuous family of positive eigenvalues that lie in a neighborhood of the origin and which are not simple (their multiplicity being at least two). Furthermore, we obtain

$$
\inf _{u \in W_{0}^{1, p(x)}(\Omega) \backslash\{0\}} \frac{\int_{\Omega}|\nabla u|^{p(x)} d x}{\int_{\Omega}\left(|u|^{a}+|u|^{b}\right) d x}=0 .
$$

4. Proof of Theorem 1. Let $E$ denote the generalized Sobolev space $W_{0}^{1, p(x)}(\Omega)$. The energy functional corresponding to problem (6) is defined as $J: E \rightarrow \mathbb{R}$,

$$
J(u)=\int_{\Omega} \frac{1}{p(x)}|\nabla u|^{p(x)} d x-\frac{A}{a} \int_{\Omega}|u|^{a} d x-\frac{B}{b} \int_{\Omega}|u|^{b} d x .
$$

Arguments similar to those used in $[\mathbf{1 1}]$ ensure that $J \in C^{1}(E, \mathbb{R})$ with

$$
\left\langle J^{\prime}(u), v\right\rangle=\int_{\Omega}|\nabla u|^{p(x)-2} \nabla u \nabla v d x-A \int_{\Omega}|u|^{a-2} u v d x-B \int_{\Omega}|u|^{b-2} u v d x,
$$

for all $u, v \in E$. Thus the weak solutions of (6) are exactly the critical points of $J$. We shall prove that the functional $J$ possesses two distinct critical points using as main tools the Mountain Pass Theorem (see, e.g. [2]) and Ekeland's Variational Principle (see, e.g. [8]).

LEMMA 1. The following assertions hold.

(i) There exist $\rho>0$ and $\alpha>0$ such that

$$
J(u) \geq \alpha>0, \quad \forall u \in E \text { with }\|u\|=\rho .
$$

(ii) There exists $\psi \in$ E such that

$$
\lim _{t \rightarrow \infty} J(t \psi)=-\infty
$$

(iii) There exists $\varphi \in E$ such that $\varphi \geq 0, \varphi \neq 0$ and

$$
J(t \varphi)<0
$$

for $t>0$ small enough. 
Proof. (i) It is clear that $E$ is continuously embedded in $L^{a}(\Omega)$ and in $L^{b}(\Omega)$. Thus there exist two positive constants $c_{1}$ and $c_{2}$ such that

$$
\int_{\Omega}|u|^{a} d x \leq c_{1}\|u\|^{a}, \quad \forall u \in E,
$$

and

$$
\int_{\Omega}|u|^{b} d x \leq c_{2}\|u\|^{b}, \quad \forall u \in E .
$$

Using relations (8), (9) and (5) we deduce that for any $u \in E$ with $\|u\|=1$ we have

$$
J(u) \geq \frac{1}{p^{+}}-A \frac{1}{a} c_{1}-B \frac{1}{b} c_{2} .
$$

Taking

$$
\lambda=\min \left\{\frac{a}{4 p^{+} c_{1}}, \frac{b}{4 p^{+} c_{2}}\right\}
$$

and providing that $A, B \in(0, \lambda)$ we obtain

$$
J(u) \geq \frac{1}{2 p^{+}}, \quad \forall u \in E \text { with }\|u\|=1 .
$$

The first part of Lemma 1 is proved.

(ii) Let $\psi \in C_{0}^{\infty}(\Omega), \psi \geq 0, \psi \neq 0$ and $t>1$. We have

$$
\begin{aligned}
J(t \psi) & =\int_{\Omega} \frac{t^{p(x)}}{p(x)}|\nabla \psi|^{p(x)} d x-\frac{A}{a} t^{a} \int_{\Omega}|\psi|^{a} d x-\frac{B}{b} t^{b} \int_{\Omega}|\psi|^{b} d x \\
& \leq \frac{t^{p^{+}}}{p^{-}} \int_{\Omega}|\nabla \psi|^{p(x)} d x-\frac{B}{b} t^{b} \int_{\Omega}|\psi|^{b} d x .
\end{aligned}
$$

Since $b>p^{+}$we deduce that $\lim _{t \rightarrow \infty} J(t \psi)=-\infty$ and (ii) is proved.

(iii) Let $\varphi \in C_{0}^{\infty}(\Omega), \varphi \geq 0, \varphi \neq 0$ and $t \in(0,1)$. We have

$$
\begin{aligned}
J(t \varphi) & =\int_{\Omega} \frac{t^{p(x)}}{p(x)}|\nabla \varphi|^{p(x)} d x-\frac{A}{a} t^{a} \int_{\Omega}|\varphi|^{a} d x-\frac{B}{b} t^{b} \int_{\Omega}|\varphi|^{b} d x \\
& \leq \frac{t^{p^{-}}}{p^{-}} \int_{\Omega}|\nabla \varphi|^{p(x)} d x-\frac{A}{a} t^{a} \int_{\Omega}|\varphi|^{a} d x<0
\end{aligned}
$$

for $t<\delta^{1 /\left(p^{-}-a\right)}$ with

$$
0<\delta<\min \left\{1, \frac{\frac{A}{a} p^{+} \int_{\Omega}|\varphi|^{a} d x}{\int_{\Omega}|\nabla \varphi|^{p(x)} d x}\right\} .
$$

It follows that (iii) is proved.

The proof of Lemma 1 is complete.

Proof of Theorem 1. Let $\lambda>0$ be defined as in (10) and $A \in(0, \lambda), B \in(0, \lambda)$. Using Lemma 1 (i) and (ii) and the Mountain Pass Theorem (see, e.g. [2]) we deduce 
the existence of a sequence $\left\{u_{n}\right\}$ in $E$ such that

$$
J\left(u_{n}\right) \rightarrow \bar{c}>0 \quad \text { and } \quad J^{\prime}\left(u_{n}\right) \rightarrow 0 \text { in } E^{\star} .
$$

We prove that $\left\{u_{n}\right\}$ is bounded in $E$. Assume the contrary. Then, passing eventually to a subsequence, still denoted by $\left\{u_{n}\right\}$, we may assume that $\left\|u_{n}\right\| \rightarrow \infty$ as $n \rightarrow \infty$. Thus we may consider that $\left\|u_{n}\right\|>1$, for any integer $n$. Relations (11) and (4) imply that for $n$ large enough we have

$$
\begin{aligned}
\bar{c}+1+\left\|u_{n}\right\| & \geq J\left(u_{n}\right)-\frac{1}{b}\left\langle J^{\prime}\left(u_{n}\right), u_{n}\right\rangle \\
& =\int_{\Omega} \frac{1}{p(x)}\left|\nabla u_{n}\right|^{p(x)} d x-\frac{A}{a} \int_{\Omega}\left|u_{n}\right|^{a} d x-\frac{B}{b} \int_{\Omega}\left|u_{n}\right|^{b} d x \\
& -\frac{1}{b} \int_{\Omega}\left|\nabla u_{n}\right|^{p(x)} d x+\frac{A}{b} \int_{\Omega}\left|u_{n}\right|^{a} d x+\frac{B}{b} \int_{\Omega}\left|u_{n}\right|^{b} d x \\
& \geq\left(\frac{1}{p^{+}}-\frac{1}{b}\right)\left\|u_{n}\right\|^{p^{-}}+A\left(\frac{1}{b}-\frac{1}{a}\right) \int_{\Omega}\left|u_{n}\right|^{a} d x .
\end{aligned}
$$

Since $a, p^{+}<b$ and relation (8) holds true, we deduce the existence of a positive constant $c_{3}$ such that

$$
\bar{c}+1+\left\|u_{n}\right\| \geq\left(\frac{1}{p^{+}}-\frac{1}{b}\right)\left\|u_{n}\right\|^{p^{-}}-c_{3}\left\|u_{n}\right\|^{a},
$$

for $n$ large enough. Dividing the above inequality by $\left\|u_{n}\right\|^{p^{-}}$and passing to the limit as $n \rightarrow \infty$ we obtain a contradiction. It follows that $\left\{u_{n}\right\}$ is bounded in $E$. Thus, there exists $u_{1} \in E$ such that passing to a subsequence, still denoted by $\left\{u_{n}\right\}$, it converges weakly to $u_{1}$ in $E$. Since $a, b<\frac{N p^{-}}{N-p^{-}}$we deduce that $E$ is compactly embedded in $L^{a}(\Omega)$ and $L^{b}(\Omega)$ and it follows that $\left\{u_{n}\right\}$ converges strongly to $u_{1}$ in $L^{a}(\Omega)$ and $L^{b}(\Omega)$. On the other hand, relation (11) yields

$$
\lim _{n \rightarrow \infty}\left\langle J^{\prime}\left(u_{n}\right), u_{n}-u_{1}\right\rangle=0
$$

Using the above information we find that

$$
\lim _{n \rightarrow \infty} \int_{\Omega}\left|\nabla u_{n}\right|^{p(x)-2} \nabla u_{n} \nabla\left(u_{n}-u_{1}\right) d x=0 .
$$

Relation (12) and the fact that $\left\{u_{n}\right\}$ converges weakly to $u_{1}$ in $E$ enable us to apply Theorem 3.1 in [11] in order to obtain that $\left\{u_{n}\right\}$ converges strongly to $u_{1}$ in $E$. Then, since $J \in C^{1}(E, \mathbb{R})$ and relation (11) holds true we conclude that

$$
J\left(u_{1}\right)=\bar{c} \quad \text { and } \quad J^{\prime}\left(u_{1}\right)=0 .
$$

It follows that $u_{1}$ is a nontrivial weak solution for problem (6).

We prove now that there exists a second weak solution $u_{2} \in E$ such that $u_{2} \neq u_{1}$. By Lemma 1 (i) it follows that on the boundary of the unit ball, centered at the origin in $E$ and denoted by $B_{1}(0)$, we have

$$
\inf _{\partial B_{1}(0)} J>0 .
$$


On the other hand, by Lemma 1 (iii) there exists $\varphi \in E$ such that $J(t \varphi)<0$, for all $t>0$ small enough. Moreover, relations (8), (9) and (4) imply that, for any $u \in B_{1}(0)$, the inequality

$$
J(u) \geq \frac{1}{p^{+}}\|u\|^{p^{+}}-\frac{A}{a} c_{1}\|u\|^{a}-\frac{B}{b} c_{2}\|u\|^{b}
$$

holds and we deduce that

$$
-\infty<\underline{c}:=\inf _{B_{1}(0)} J<0 .
$$

We now let $0<\epsilon<\inf _{\partial B_{1}(0)} J-\inf _{B_{1}(0)} J$. Apply Ekeland's Variational Principle for the functional $J: \overline{B_{1}(0)} \rightarrow \mathbb{R}$, (see [8]). There exists $u_{\epsilon} \in \overline{B_{1}(0)}$ such that

$$
\begin{aligned}
& J\left(u_{\epsilon}\right)<\inf _{\overline{B_{1}}(0)} J+\epsilon, \\
& J\left(u_{\epsilon}\right)<J(u)+\epsilon \cdot\left\|u-u_{\epsilon}\right\|, \quad u \neq u_{\epsilon} .
\end{aligned}
$$

Since

$$
J\left(u_{\epsilon}\right) \leq \inf _{B_{1}(0)} J+\epsilon \leq \inf _{B_{1}(0)} J+\epsilon<\inf _{\partial B_{1}(0)} J
$$

it follows that $u_{\epsilon} \in B_{1}(0)$. Now, we define $I: \overline{B_{1}(0)} \rightarrow \mathbb{R}$ by $I(u)=J(u)+\epsilon \cdot\left\|u-u_{\epsilon}\right\|$. It is clear that $u_{\epsilon}$ is a minimum point of $I$ and thus

$$
\frac{I\left(u_{\epsilon}+t \cdot v\right)-I\left(u_{\epsilon}\right)}{t} \geq 0
$$

for a small $t>0$ and $v \in B_{1}(0)$. The relation above yields

$$
\frac{J\left(u_{\epsilon}+t \cdot v\right)-J\left(u_{\epsilon}\right)}{t}+\epsilon \cdot\|v\| \geq 0
$$

Letting $t \rightarrow 0$ it follows that $\left\langle J^{\prime}\left(u_{\epsilon}\right), v\right\rangle+\epsilon \cdot\|v\|>0$ and we infer that $\left\|J^{\prime}\left(u_{\epsilon}\right)\right\| \leq \epsilon$.

We deduce that there exists a sequence $\left\{w_{n}\right\} \subset B_{1}(0)$ such that

$$
J\left(w_{n}\right) \rightarrow \underline{c} \text { and } \quad J^{\prime}\left(w_{n}\right) \rightarrow 0 .
$$

Using the same arguments as in the case of solution $u_{1}$ we can prove that $\left\{w_{n}\right\}$ converges strongly to $u_{2}$ in $E$. Moreover, since $J \in C^{1}(E, \mathbb{R})$, by relation (15) it follows that

$$
J\left(u_{2}\right)=\underline{c} \text { and } \quad J^{\prime}\left(u_{2}\right)=0 .
$$

Thus, $u_{2}$ is also a nontrivial weak solution for problem (6).

Finally, we point out the fact that $u_{1} \neq u_{2}$ since

$$
J\left(u_{1}\right)=\bar{c}>0>\underline{c}=J\left(u_{2}\right) .
$$

The proof of Theorem 1 is complete.

AcKnowledgments. The author would like to thank Professor V. Rădulescu for proposing this problem and for numerous valuable discussions. 


\section{REFERENCES}

1. E. Acerbi and G. Mingione, Regularity results for a class of functionals with nonstandard growth, Arch. Rational Mech. Anal. 156 (2001), 121-140.

2. H. Brezis and L. Nirenberg, Remarks on finding critical points, Comm. Pure Appl. Math. 44 (1991), 939-963.

3. J. Chabrowski and $\mathrm{Y}$. Fu, Existence of solutions for $p(x)$-Laplacian problems on bounded domains, J. Math. Anal. Appl., in press (doi:10.1016/j.jmaa.2004.10.028).

4. L. Diening, Theoretical and numerical results for electrorheological fluids, Ph.D. thesis (University of Freiburg, Germany, 2002).

5. D. E. Edmunds, J. Lang and A. Nekvinda, On $L^{p(x)}$ norms, Proc. Roy. Soc. London Ser. A 455 (1999), 219-225.

6. D. E. Edmunds and J. Rákosník, Density of smooth functions in $W^{k, p(x)}(\Omega)$, Proc. Roy. Soc. London Ser. A 437 (1992), 229-236.

7. D. E. Edmunds and J. Rákosník, Sobolev embedding with variable exponent, Studia Math. 143 (2000), 267-293.

8. I. Ekeland, On the variational principle, J. Math. Anal. App. 47 (1974), 324-353.

9. X. Fan and X. Han, Existence and multiplicity of solutions for $p(x)$-Laplacian equations in $\mathbb{R}^{N}$, Nonlinear Anal. 59 (2004), 173-188.

10. X. Fan, J. Shen and D. Zhao, Sobolev Embedding Theorems for Spaces $W^{k, p(x)}(\Omega)$, J. Math. Anal. Appl. 262 (2001), 749-760.

11. X. L. Fan and Q. H. Zhang, Existence of solutions for $p(x)$-Laplacian Dirichlet problem, Nonlinear Anal. 52 (2003), 1843-1852.

12. X. Fan, Q. Zhang and D. Zhao, Eigenvalues of $p(x)$-Laplacian Dirichlet problem, J. Math. Anal. Appl. 302 (2005), 306-317.

13. X. L. Fan and D. Zhao, On the Spaces $L^{p(x)}(\Omega)$ and $W^{m, p(x)}(\Omega)$, J. Math. Anal. Appl., 263 (2001), 424-446.

14. T. C. Halsey, Electrorheological fluids, Science 258 (1992), 761-766.

15. O. Kováčik and J. Rákosník, On spaces $L^{p(x)}$ and $W^{1, p(x)}$, Czechoslovak Math. J. 41 (1991), 592-618.

16. M. Mihăilescu and V. Rădulescu, A multiplicity result for a nonlinear degenerate problem arising in the theory of electrorheological fluids, Proc. Roy. Soc. London Ser. A, in press, (doi:10.1098/rspa.2005.1633).

17. J. Musielak, Orlicz spaces and modular spaces, Lecture Notes in Mathematics, No. 1034 (Springer-Verlag, Berlin, 1983).

18. H. Nakano, Modulared semi-ordered linear spaces (Maruzen Co., Ltd., Tokyo, 1950).

19. W. Orlicz, Über konjugierte Exponentenfolgen, Studia Math. 3 (1931), 200-212.

20. M. Ruzicka, Flow of shear dependent electrorheological fluids, C. R. Acad. Sci. Paris Ser. I Math. 329 (1999), 393-398.

21. M. Ruzicka, Electrorheological fluids modeling and mathematical theory (SpringerVerlag, 2002).

22. I. Sharapudinov, On the topology of the space $L^{p(t)}([0 ; 1])$, Matem. Zametki 26 (1978), 613-632.

23. I. Tsenov, Generalization of the problem of best approximation of a function in the space $L^{s}$, Uch. Zap. Dagestan Gos. Univ. 7 (1961), 25-37.

24. W. M. Winslow, Induced fibration of suspensions, J. Appl. Phys. 20 (1949), 1137-1140.

25. V. Zhikov, Averaging of functionals in the calculus of variations and elasticity, Math. USSR Izv. 29 (1987), 33-66.

26. V. Zhikov, On passing to the limit in nonlinear variational problem, Math. Sb. 183 (1992), 47-84. 